\title{
Cell-free microRNAs in urine as diagnostic and prognostic biomarkers of bladder cancer
}

\author{
SEOK JOONG YUN ${ }^{1}$, PILDU JEONG ${ }^{1}$, WON-TAE KIM ${ }^{1}$, TAE HWAN KIM ${ }^{2}$, YOUNG-SUK LEE ${ }^{3}$, \\ PHIL HYUN SONG ${ }^{1,4}$, YUNG-HYUN CHOI ${ }^{5}$, ISAAC YI KIM ${ }^{6}$, SUNG-KWON MOON $^{7}$ and WUN-JAE KIM ${ }^{1}$ \\ ${ }^{1}$ Department of Urology, Chungbuk National University College of Medicine, Cheongju; ${ }^{2}$ Kyungpook National University \\ School of Medicine, Daegu; ${ }^{3}$ Samsung Changwon Hospital, Sungkyunkwan University School of Medicine, Changwon; \\ ${ }^{4}$ Yeungnam University College of Medicine, Daegu; ${ }^{5}$ Department of Biochemistry, Dongeui University College of \\ Oriental Medicine, Busan, Republic of Korea; ${ }^{6}$ Section of Urologic Oncology, The Cancer Institute of New Jersey, \\ Robert Wood Johnson Medical School, New Brunswick, NJ, USA; ${ }^{7}$ Department of Biotechnology, \\ Chungju National University, Chungju, Republic of Korea
}

Received June 30, 2012; Accepted August 20, 2012

DOI: 10.3892/ijo.2012.1622

\begin{abstract}
RNAs are small, non-coding RNAs that play important roles in various biological processes. The aims of our study were to investigate whether cell-free miRNAs can be measured in urine samples and might be an accurate biomarker of bladder cancer. Datasets of GSE20418 and GSE19717 were used for analysis, and two miRNAs, miR-145 and miR-200a, were selected for study. A total of 207 patients with primary transitional cell carcinoma of the urinary bladder and 144 healthy normal controls were enrolled. Using quantitative PCR, the levels of miR-145 and miR-200a in urine were measured and compared with the clinicopathological features of bladder cancer. According to our experiments, cell-free miRNAs were present in urine and were stable. Assessment of miR-145 levels was able to distinguish bladder cancer patients from non-cancer controls $(77.8 \%$ sensitivity and $61.1 \%$ specificity for NMIBC, AUC $0.729 ; 84.1$ and $61.1 \%$ for MIBC, respectively, AUC $0.790)$ and showed good correlation with grade $(p=0.048)$. In addition, miR-200a was shown to be an independent predictor of NMIBC recurrence by multivariate analysis (OR 0.449 ,
\end{abstract}

Correspondence to: Professor Sung-Kwon Moon, Department of Biotechnology, Chungju National University, 123 Geomdan-ri Iryu-myeon, Chungju, Chungbuk, 380-702, Republic of Korea E-mail: sumoon66@dreamwiz.com

Professor Wun-Jae Kim, Department of Urology, Chungbuk National University, College of Medicine and Institute for Tumor Research, 62 Kaeshin-dong, Heungduk-gu, Cheongju 361-763, Republic of Korea E-mail:wjkim@chungbuk.ac.kr

Abbreviations: miRNA, microRNA; TUR, transurethral resection; NMIBC, non-muscle invasive bladder cancer; MIBC, muscle invasive bladder cancer; AUC, area under the curve

Key words: microRNAs, bladder cancer, urine
95\% CI 0.239-0.842, $\mathrm{p}=0.013$ ). A higher risk of recurrence was observed among patients with a lower miR-200a level compared to patients with higher miR-200a levels (log-rank test, $\mathrm{p}=0.040$ ). Urinary cell-free miRNAs show promise as noninvasive biomarkers for diagnosis and recurrence of bladder cancer.

\section{Introduction}

Surveillance strategies for bladder cancer recurrence have historically relied on the diagnostic combination of cystoscopy and urinary cytology. However, the cystoscopic approach is costly, invasive and uncomfortable. Urinary cytology is a preferable technique for the diagnosis of bladder tumors because of its high specificity; however, it has low sensitivity. For these reasons, many new urine-based tests for urinary bladder cancer have been developed, and screens for bladder tumor antigen (BTA), nuclear matrix protein 22 (NMP22), urine fibrin fibrinogen degradation products (FDP), ImmunoCyt and FISH (UroVysion) have all been approved for clinical use $(1,2)$. However, the specificities of these new urine markers are low in comparison with urinary cytology, although they have higher sensitivities. Thus, none of the currently identified urine markers can replace cystoscopy or urinary cytology (3).

miRNAs are small, nonprotein-coding RNA regulators involved in numerous biological and developmental processes $(4,5)$. Cumulative evidence suggests that the dysregulation of miRNA plays an important role in many human disorders, including cancer. Approximately 50\% of human miRNAs are encoded in genomic regions that are frequently altered in cancer (6-8). Recently, miRNAs have emerged as highly tissue-specific biomarkers with potential clinical applicability, not only as diagnostic markers but also as prognostic predictors for numerous cancers.

Numerous recent studies have explored circulating cell-free miRNAs and provided evidence that miRNAs exist in a stable form in various body fluids, such as blood, urine, saliva, and peritoneal fluid (9). Understanding the characteristics of bladder cancer, urine is a desirable biomarker source because 
the cancer directly contacts the urine and it is convenient to collect. However, few studies have examined the detection of urinary miRNAs as diagnostic or prognostic markers of bladder cancer $(10,11)$.

In the current study, we investigated whether cell-free miRNAs could be detected in urine samples of bladder cancer patients and healthy controls, and validated the stability of the miRNAs and the reproducibility of their detection under various conditions. Finally, we compared the expression of urinary cell-free miRNAs with clinical outcomes and assessed the value of using miRNAs as diagnostic and prognostic biomarkers of bladder cancer.

\section{Materials and methods}

Selection of putative target miRNAs. A review of the literature was performed using the National Library of Medicine database (http://www.pub-med.gov) to extract miRNA signatures significantly associated with bladder cancer. Datasets of GSE20418 and GSE19717 were used for analysis, and a total of 21 miRNAs were selected for the current study (12). These extracted miRNA signatures were previously reported to be significantly associated with urological cancers and were obtained by gene expression profiling or non-coding RNA profiling studies using microarrays. Based on preliminary miRNA expression analysis in urine samples, miR-145 and miR-200a were selected for the current study.

Study population and samples. A total of 207 patients with primary urothelial carcinoma of the urinary bladder and 144 healthy controls who visited the hospital for a medical check-up were enrolled in the study. Controls were selected with similar age and gender proportions to the cancer patients, and subjects were screened to ensure that they were within the normal range of all laboratory findings and had no history of cancer. The collection and analysis of all samples was approved by the Institutional Review Board of Chungbuk National University, and written informed consent was obtained from each subject (IRB approval number 2006-01-001). Urine samples were collected in the morning and stored at $4^{\circ} \mathrm{C}$, then centrifuged at 25,000 rpm for $15 \mathrm{~min}$. Each supernatant and sediment was aliquoted into Eppendorf tubes and stored at $-20^{\circ} \mathrm{C}$ until use. All primary tumor samples were obtained from patients who underwent TUR or radical cystectomy and were histologically verified to have urothelial carcinoma. In cases of NMIBC, patients who had a T1 tumor, multiple tumors, large tumors ( $>3 \mathrm{~cm}$ in diameter), or high grade Ta NMIBC received one cycle of intravesical treatment (BCG or mitomycin-C). Response to treatment was assessed by cystoscopy and urinary cytology. Patients who were free of disease within three months after treatment were assessed every three months for the first two years, and then every six months thereafter. In cases of MIBC, patients with clinically localized or locally advanced tumors and good ECOG performance status (0 or 1) underwent radical cystectomy and complete pelvic lymph node dissection. Patients who were not eligible for radical cystectomy due to metastatic disease, poor life expectancy, or poor ECOG performance status $(\geq 2)$ underwent TUR or biopsy for histopathological diagnosis. Patients with pT3, pT4 or nodepositive disease based on the analysis of radical cystectomy specimens, or with metastatic disease but good performance status, received at least four cycles of cisplatin-based chemotherapy. Patients who refused or did not complete an imaging work-up such as a CT scan or MRI at least once every three months to evaluate their response were excluded from analysis. Tumors were staged and graded according to the 2002 TNM classification and the 1973 WHO grading system, respectively. Recurrence was defined as recurrence of primary NMIBC at a lower or the same pathological stage, and progression was defined as disease with a higher TNM stage upon relapse of NMIBC. In cases of MIBC, progression was defined as local regional recurrence or a new distant metastasis in the cystectomized group and $a \geq 20 \%$ increase in the mass of the primary tumor or a new distant metastasis in the non-cystectomized group.

Purification ofmiRNA from urine. The Genolution Urine miRNA Purification Kit (Genolution Pharmaceuticals Inc., Seoul, Korea) was used to purify urine samples. Urine sample supernatant $(500 \mu \mathrm{l})$ was added to each tube containing Genolution proprietary miRNA separation solution and vortexed for $20 \mathrm{sec}$. Next, $200 \mu \mathrm{l}$ of chloroform was added and the samples vortexed for $10 \mathrm{sec}$, after which they were centrifuged at 13,000 rpm for $10 \mathrm{~min}$ at $4^{\circ} \mathrm{C}$. A $600 \mu \mathrm{l}$ fraction from the top aqueous phase was taken and transferred into a new $1.5-\mathrm{ml}$ tube, and $0.8 \mathrm{ml}$ of isopropanol was added, followed by centrifugation for $5 \mathrm{~min}$ at $13,000 \mathrm{rpm}$ and $4^{\circ} \mathrm{C}$. After removing the aqueous solution, $1 \mathrm{ml}$ of $70 \%$ ethanol was added and the sample was again centrifuged for $2 \mathrm{~min}$ at $13,000 \mathrm{rpm}$ and $4^{\circ} \mathrm{C}$. After removing the ethanol, the pellet was dissolved in $30 \mu \mathrm{l}$ RNase free water and stored at $-80^{\circ} \mathrm{C}$ until use. A fixed concentration of microRNA $(5 \mathrm{ng} / \mu \mathrm{l})$ from a given volume of starting urine was used as the input into the reverse transcription reaction.

Reverse transcription of miRNA. The miScript Reverse Transcription Kit (Qiagen Korea, Seoul, South Korea) was used for reverse transcription of the miRNAs. After mixing with template RNA, 5X miScript buffer, miScript Reverse Transcriptase mix and RNase-free water in a final volume of $20 \mu \mathrm{l}$, the mixture was centrifuged briefly and incubated for $60 \mathrm{~min}$ at $37^{\circ} \mathrm{C}$. To inactivate the miScript Reverse Transcriptase mix, the samples were incubated for $5 \mathrm{~min}$ at $95^{\circ} \mathrm{C}$ and placed on ice.

Real-time PCR detection of miRNA. To quantify miRNA expression, real-time PCR amplification was performed with a Rotor-Gene $^{\mathrm{TM}}$ 6000. Real-time PCR assays were performed in microreaction tubes (Corbett Research, Mortlake, Australia) using the miScript PCR Starter Kit (Qiagen Korea, Seoul, Korea).

For amplification of the target miRNAs, forward primers were designed for miR-145 (5'- GTC CAG TTT TCC CAG GAA TCC CT- 3') and miR-200a (5'-TAA CAC TGT CTG GTA ACG AT GT- $3^{\prime}$ ). After thawing the reagents and template cDNA, the PCR reaction was carried out in a final volume of $20 \mu \mathrm{l}$ containing $10 \mu \mathrm{l} 2 \mathrm{X}$ QuantiTect SYBR-Green PCR Master mix, $2 \mu 1$ 10X miScript Universal Primer, $2 \mu 110$ pmol forward primer, $2 \mu \mathrm{l}$ template cDNA and RNase-free water. Real-time PCR conditions were: one cycle of initial activation for $15 \mathrm{~min}$ at $95^{\circ} \mathrm{C}$, followed by 50 cycles of $15 \mathrm{sec}$ at $94^{\circ} \mathrm{C}$ for denaturation, annealing for $30 \mathrm{sec}$ at $55^{\circ} \mathrm{C}$ and extension for $30 \mathrm{sec}$ at $70^{\circ} \mathrm{C}$. The melting program was performed at $70^{\circ} \mathrm{C}$ to $99^{\circ} \mathrm{C}$ at a heating rate of $1^{\circ} \mathrm{C}$ per $5 \mathrm{sec}$. Spectral data were captured and 
A

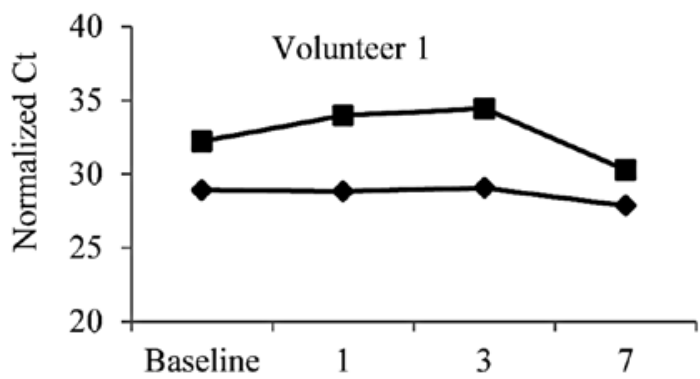

Number of freeze-thaw cycles

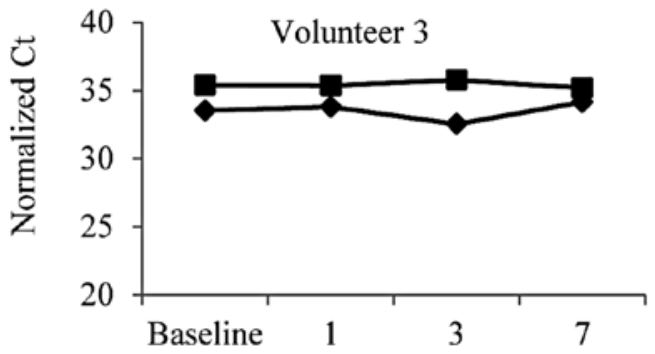

Number of freeze-thaw cycles

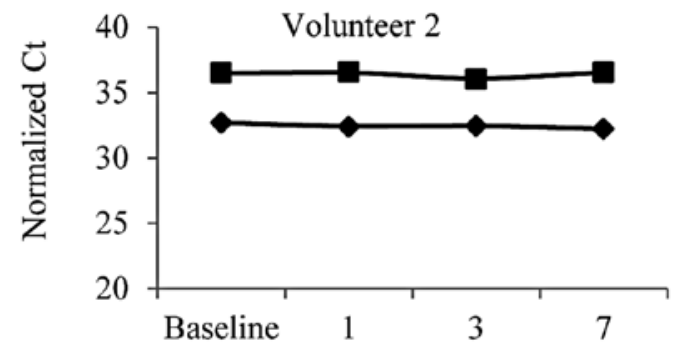

Number of freeze-thaw cycles

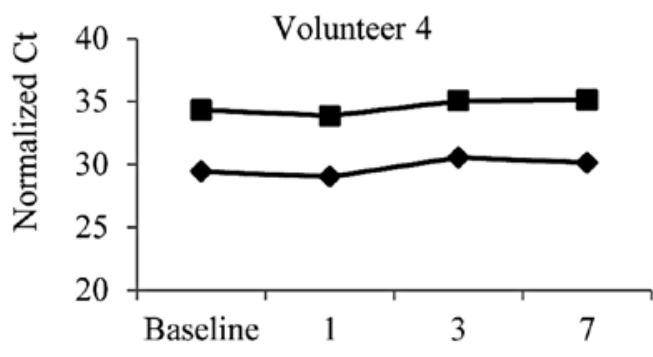

Number of freeze-thaw cycles

B

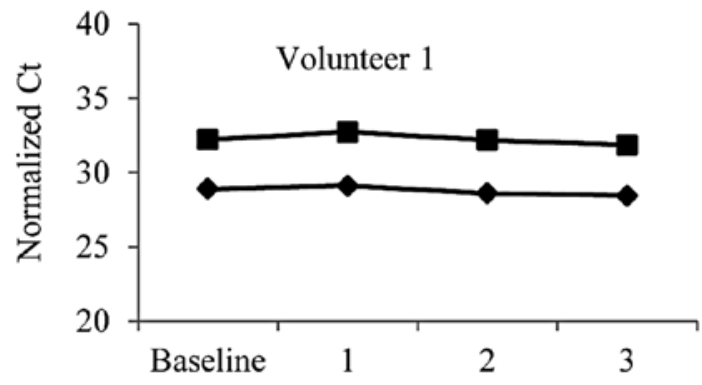

Incubation days at room temperature

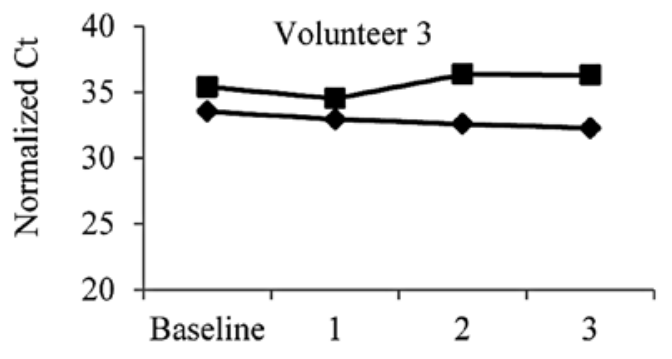

Incubation days at room temperature

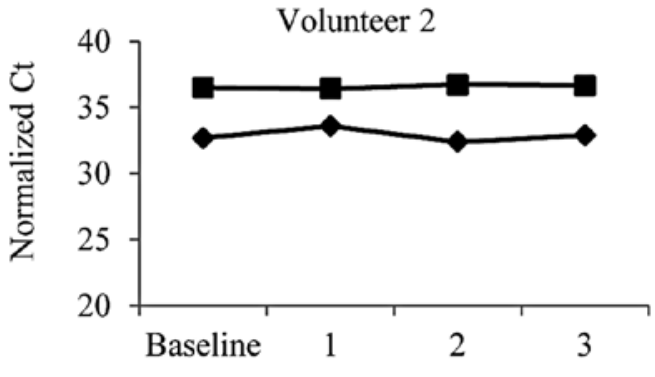

Incubation days at room temperature

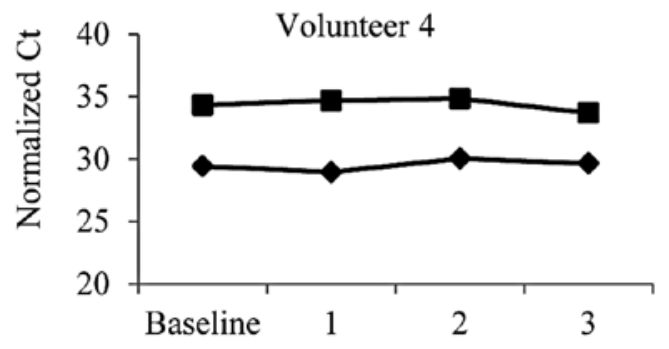

Incubation days at room temperature

Figure 1. Validation of the stability of cell-free miRNA in urine. Urine samples from four healthy volunteers (A) underwent seven cycles of freezing and thawing or (B) were incubated at room temperature for $72 \mathrm{~h}$. The levels of miRNAs were not significantly changed in any of the experimental samples, indicating that cell-free miRNAs in urine exist in a stable form.

analyzed using Rotor-Gene Real-Time Analysis Software 6.0, Build 14. All samples were run in triplicate.

For accurate and reproducible results by real-time PCR, it is necessary to normalize the amount of target miRNA using a suitable endogenous reference RNA. This approach is known as relative quantification. In this experiment, U6 was used as a control for the normalization of real-time PCR results in miRNA quantification studies using the miScript PCR System. U6 was analyzed in parallel as an endogenous RNA reference gene and all data were normalized to U6 expression. 
Table I. Baseline clinical and pathological features of patients with bladder cancer.

\begin{tabular}{|c|c|c|}
\hline Variables & $\begin{array}{l}\text { No. of patients } \\
(\%)\end{array}$ & $\begin{array}{c}\text { No. of controls } \\
(\%)\end{array}$ \\
\hline Number & 207 & 144 \\
\hline Mean age $\pm S D$ & $63.52 \pm 12.64$ & $63.79 \pm 12.65$ \\
\hline \multicolumn{3}{|l|}{ Gender } \\
\hline Male & 165 (79.7) & 114 (79.2) \\
\hline Female & $42(20.3)$ & $30(20.8)$ \\
\hline \multicolumn{3}{|l|}{ Grade } \\
\hline G1 & $53(25.6)$ & \\
\hline $\mathrm{G} 2$ & $86(41.4)$ & \\
\hline G3 & $68(32.9)$ & \\
\hline \multicolumn{3}{|l|}{$\mathrm{T}$ stage } \\
\hline $\mathrm{Ta}$ & $64(30.9)$ & \\
\hline $\mathrm{T} 1$ & $74(35.7)$ & \\
\hline $\mathrm{T} 2$ & $28(13.5)$ & \\
\hline $\mathrm{T} 3$ & $22(10.6)$ & \\
\hline $\mathrm{T} 4$ & $19(9.2)$ & \\
\hline \multicolumn{3}{|l|}{$\mathrm{N}$ stage } \\
\hline No & $184(88.9)$ & \\
\hline $\mathrm{N}(1-3)$ & $23(11.1)$ & \\
\hline \multicolumn{3}{|l|}{ M stage } \\
\hline M0 & $197(95.2)$ & \\
\hline M1 & $10(4.8)$ & \\
\hline $\begin{array}{l}\text { Median follow-up } \\
\text { period (months) }\end{array}$ & $41.3 \pm 34.3$ & \\
\hline
\end{tabular}

Statistical analysis. Since miRNA expression was highly skewed despite attempted data transformation, the Kruskal-Wallis test or the Mann-Whitney U test was used to compare miRNAs levels between groups. Receiver operating characteristics (ROC) curves were used to evaluate the diagnostic performance of miR-145 and miR-200a. The prognostic value of miR-200a for recurrence was analyzed by multivariate Cox proportional hazard regression models, and the optimal cutoff point for miR-200a was determined using a ROC curve analysis that yielded the highest combined sensitivity and specificity for recurrence. The Kaplan-Meier method was used to estimate the time to recurrence, and differences were assessed using log-rank statistics. Statistical analysis was performed using SPSS 12.0 software (SPSS Inc., Chicago, IL, USA), and a p-value of $<0.05$ was considered statistically significant.

\section{Results}

Baseline characteristics. The baseline characteristics of enrolled patients and controls are listed in Table I. The mean age of patients was $63.52 \pm 12.64$ with 165 males and 42 females, and the controls were $63.79 \pm 12.65$ with 114 males and 30 females. The number of patients in grade G1, G2 and G3 were 53, 86 and 68, and the number of patients
Table II. Levels of urinary miR-145 and miR-200a grouped according to the clinopathological features of bladder cancer.

\begin{tabular}{lcccc}
\hline Variables & miR-145 & P-value & miR-200a & P-value \\
\hline Grade & & $0.048^{\mathrm{a}}$ & & $0.375^{\mathrm{a}}$ \\
G1 & $18.10 \pm 2.25$ & & $12.45 \pm 3.17$ & \\
G2 & $17.25 \pm 2.31$ & & $12.56 \pm 2.82$ & \\
G3 & $17.26 \pm 2.05$ & & $11.96 \pm 2.93$ & \\
T stage & & $0.183^{\mathrm{a}}$ & & $0.256^{\mathrm{a}}$ \\
Ta & $17.59 \pm 2.59$ & & $12.93 \pm 2.97$ & \\
T1 & $17.80 \pm 1.74$ & & $12.18 \pm 2.81$ & \\
T2 & $17.32 \pm 2.30$ & & $12.37 \pm 2.96$ & \\
T3 & $17.19 \pm 2.34$ & & $11.63 \pm 3.39$ & \\
T4 & $16.37 \pm 2.27$ & & $11.84 \pm 2.57$ & \\
N stage & & $0.715^{\mathrm{b}}$ & & $0.931^{\mathrm{b}}$ \\
N0 & $17.52 \pm 2.19$ & & $12.35 \pm 2.93$ & \\
N1-3 & $17.07 \pm 2.51$ & & $12.31 \pm 3.03$ & \\
M stage & & $0.137^{\mathrm{b}}$ & & $0.193^{\mathrm{b}}$ \\
M0 & $17.44 \pm 2.20$ & & $12.30 \pm 2.93$ & \\
M1 & $18.12 \pm 2.75$ & & $13.31 \pm 2.85$ & \\
\hline
\end{tabular}

aStatistical significance was determined by the Kruskal-Wallis test. ${ }^{\mathrm{b}}$ statistical significance was determined by the Mann-Whitey $U$ test.

in stage Ta, T1, T2, T3 and T4 were 64, 74, 28, 22 and 19, respectively. A total of $23(11.1 \%)$ patients had one or more lymph node metastasis and $10(4.8 \%)$ had distant metastasis. The median follow-up date was 41.3 months. The number of NMIBC patients was $138(66.7 \%)$, and the number of MIBC patients was $69(33.3 \%)$. Intravesical therapy was performed in $71(51.4 \%)$ of the NMIBC patients after TUR. A total of 42 $(60.9 \%)$ of the 69 MIBC patients underwent radical cystectomy, and 34 (49.3\%) received cisplatin-based chemotherapy.

Validation of miRNAs in the supernatant urine. To investigate the stability of miRNA in the supernatant urine, urine samples from four healthy volunteers were exposed to seven cycles of freezing and thawing or were incubated at room temperature for $72 \mathrm{~h}$. The levels of the miRNAs were not significantly changed in any of the experimental samples (Fig. 1), indicating that the miRNAs in urine supernatant exist in a stable form.

Comparison of miR-145 and miR-200a in controls and patients. The levels of miR-145 were significantly decreased in NMIBC and MIBC patients compared to controls $(\mathrm{p}<0.001)$. Also, MIBC patients had lower miR-145 levels than NMIBC patients $(p=0.036$; Fig. $2 A)$. The levels of miR-200a were also significantly decreased in NMIBC and MIBC patients compared to controls ( $<<0.001$; Fig. $2 B$ ). Subsequently, ROC curve analysis was performed to determine the capacity of miR-145 expression to distinguish the bladder cancer patients from the controls. The levels of miR-145 in the urine showed an area under the curve (AUC) of 0.729 with a sensitivity of $77.8 \%$ and a specificity of $61.1 \%$ for NMIBC and an AUC of 0.790 with a sensitivity of $84.1 \%$ and a specificity of 

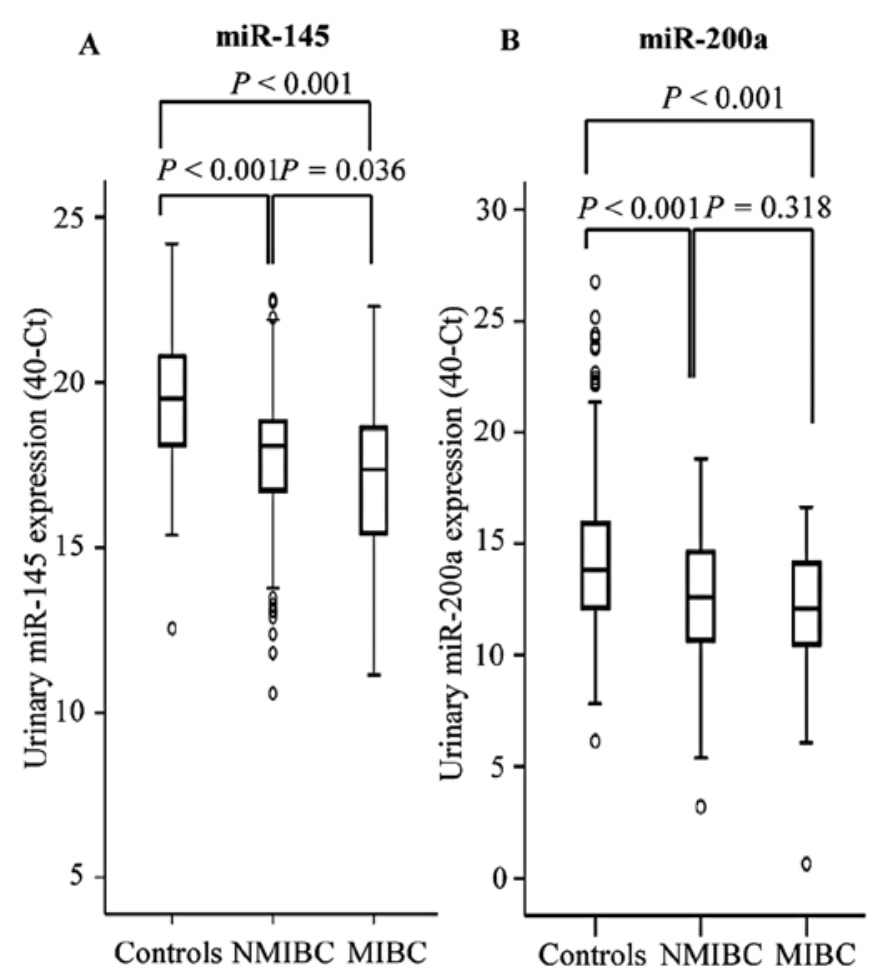

Figure 2. Comparison of urinary miRNAs between controls and bladder cancer patients. miRNA level is specified as $40-\mathrm{Ct}$, where $\mathrm{Ct}$ is the cycle threshold of the PCR reaction. Results were normalized by subtracting the U6 level in the sample. Statistical significance was determined by the Mann-Whitey U test. (A) Urinary miR-145 was lower in NMIBC $(17.71 \pm 2.16)$ and MIBC $(17.01 \pm 2.31)$ than in controls $(19.52 \pm 2.43)(\mathrm{p}<0.001$ for each). It was also lower in MIBC than NMIBC ( $\mathrm{p}=0.036)$. (B) Urinary miR-200a was lower in NMIBC $(12.51 \pm 2.93)$ and MIBC $(11.99 \pm 2.99)$ than in controls $(14.78 \pm 4.14)(\mathrm{p}<0.001$ for each).

$61.1 \%$ for MIBC (Fig. 3A and B). For miR-200a, the AUC was 0.638 with a sensitivity of $54.4 \%$ and a specificity of $65.7 \%$ for NMIBC and 0.679 with a sensitivity of $55.1 \%$ and a specificity of $72.7 \%$ for MIBC.

Comparison of miR-145 and miR-200a in predicting the clinopathological outcome of bladder cancer. Table II summarizes the association between the miRNAs and the clinicopathological parameters of bladder cancer patients. Levels of miR-145 decreased with higher grade $(p=0.048)$. Levels of miR-200a were not associated with grade or stage of bladder cancer in a statistically-significant manner. When we compared the miRNAs to clinical outcomes, miR-200a was associated with the recurrence of NMIBC (Table III). Patients who experienced recurrence of NMIBC had lower levels of miR-200a than those who had no recurrence $(11.63 \pm 3.20$ vs. $12.89 \pm 2.73, p=0.043)$. Univariate and multivariate Cox regression analysis revealed that intravesical therapy and miR-200a were the only independent predictors of NMIBC recurrence (Table IV). To assess the association between miR-200a and the risk of recurrence, Kaplan-Meier analysis was conducted in patients with NMIBC. A higher risk of recurrence was observed among patients with lower miR-200a levels compared to patients with higher miR-200a levels (log-rank test, $p=0.040$; Fig. 4).
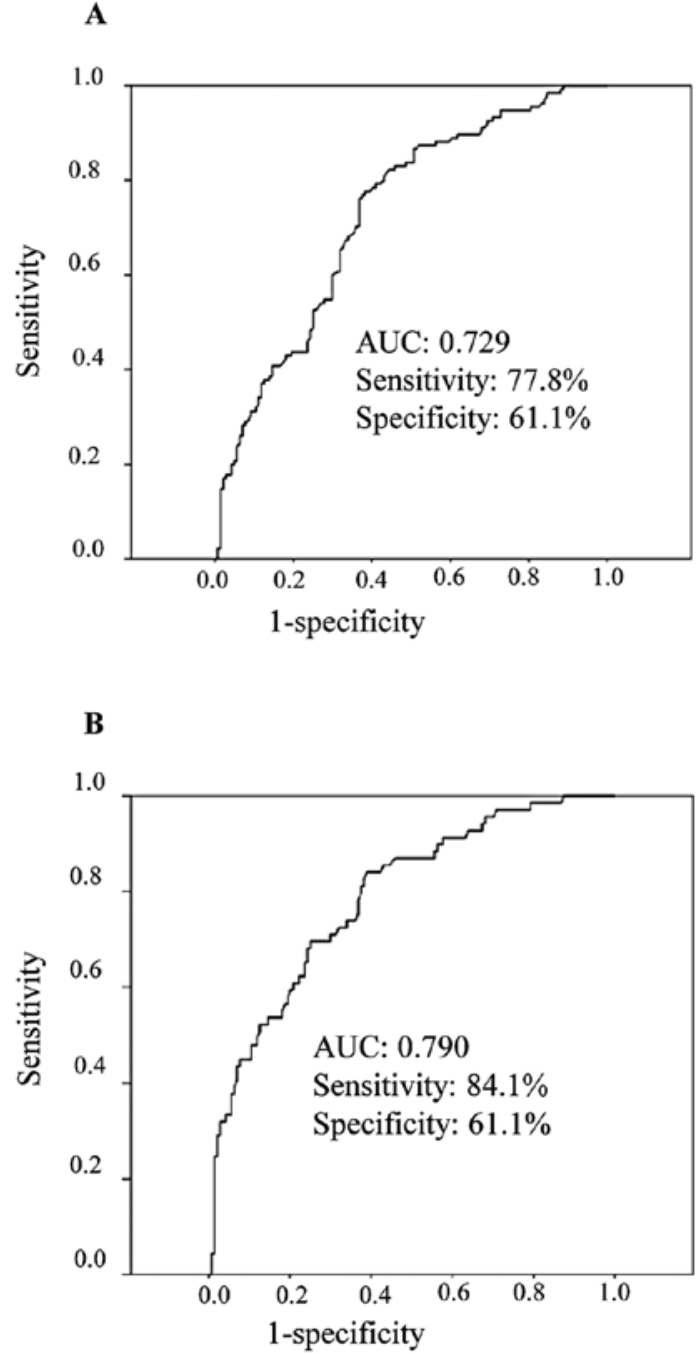

Figure 3. Diagnostic performance of urinary miR-145 detection. The receiver-operating characteristic curves for urinary miR-145 from (A) 138 NMIBC and (B) 69 MIBC patients in comparison with 144 controls.

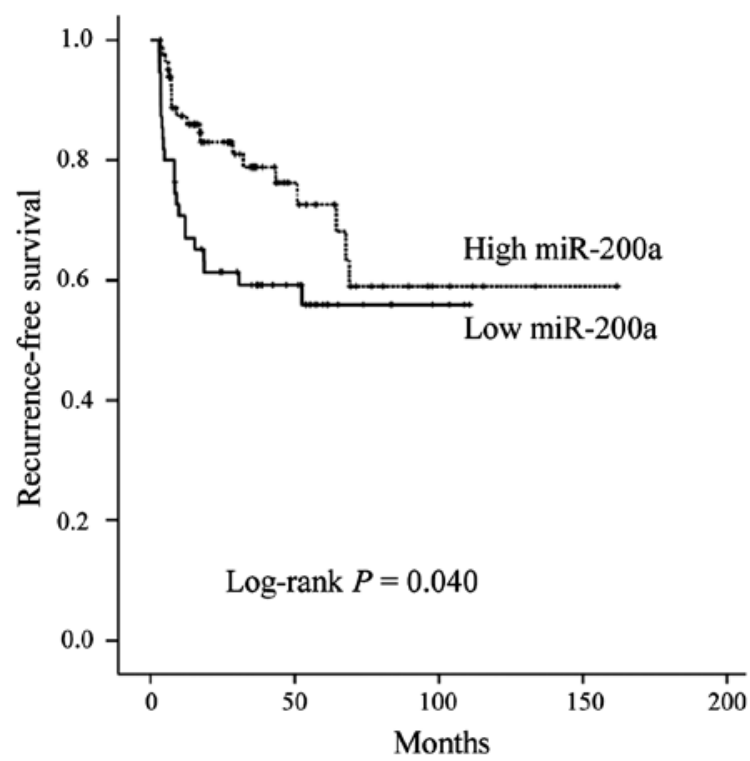

Figure 4. Kaplan-Meier estimates of the cumulative incidence of cancer recurrence in NMIBC according to the level of miR-200a. 
Table III. Levels of urinary miR-145 and miR-200a grouped according to the clinical outcomes of bladder cancer.

\begin{tabular}{|c|c|c|c|c|}
\hline Variables & $\operatorname{miR}-145$ & p-value & miR-200a & p-value \\
\hline Recurrence in NMIBC & & 0.138 & & 0.043 \\
\hline No recurrence & $17.84 \pm 2.25$ & & $12.89 \pm 2.73$ & \\
\hline Recurrence & $17.37 \pm 1.93$ & & $11.63 \pm 3.20$ & \\
\hline Progression in NMIBC & & 0.888 & & 0.436 \\
\hline No progression & $17.69 \pm 2.17$ & & $12.44 \pm 2.90$ & \\
\hline Progression & $17.84 \pm 2.13$ & & $13.05 \pm 3.15$ & \\
\hline Progression in MIBC & & 0.077 & & 0.282 \\
\hline No Progression & $17.51 \pm 2.12$ & & $12.48 \pm 2.35$ & \\
\hline Progression & $16.44 \pm 2.41$ & & $11.42 \pm 3.54$ & \\
\hline Overall survival in MIBC & & 0.825 & & 0.381 \\
\hline Alive & $17.27 \pm 2.32$ & & $12.16 \pm 3.18$ & \\
\hline Dead & $16.86 \pm 2.26$ & & $11.83 \pm 2.84$ & \\
\hline
\end{tabular}

NMIBC, non-muscle invasive bladder cancer; MIBC, muscle invasive bladder cancer.

Table IV. Univariate and multivariate Cox regression analysis for prediction of recurrence in non-muscle-invasive bladder cancer according to the miR-200a level.

\begin{tabular}{|c|c|c|c|c|}
\hline \multirow[b]{2}{*}{ Variables } & \multicolumn{2}{|l|}{ Univariate } & \multicolumn{2}{|l|}{ Multivariate } \\
\hline & HR $(95 \%$ CI $)$ & p-value & $\mathrm{HR}(95 \% \mathrm{CI})$ & p-value \\
\hline Age $(<67$ vs. $\geq 67)$ & $1.095(0.600-1.998)$ & 0.768 & $0.942(0.491-1.808)$ & 0.857 \\
\hline Sex (male vs. female) & $1.612(0.827-3.139)$ & 0.161 & $1.942(0.944-3.998)$ & 0.071 \\
\hline \multicolumn{5}{|l|}{ Number of tumors } \\
\hline Single & 1 & & 1 & \\
\hline 2 to 7 & $1.344(0.668-2.704)$ & 0.407 & $0.793(0.359-1.754)$ & 0.567 \\
\hline$\geq 8$ & $2.636(1.220-5.699)$ & 0.014 & $1.696(0.711-4.045)$ & 0.233 \\
\hline Tumor size $(<3 \mathrm{~cm}$ vs. $\geq 3 \mathrm{~cm})$ & $1.241(0.680-2.268)$ & 0.482 & $0.954(0.476-1.911)$ & 0.844 \\
\hline Stage (Ta vs. T1) & $1.045(0.569-1.920)$ & 0.887 & $0.545(0.263-1.131)$ & 0.103 \\
\hline \multicolumn{5}{|l|}{ Grade } \\
\hline G1 & 1 & & 1 & \\
\hline $\mathrm{G} 2$ & $1.231(0.621-2.438)$ & 0.552 & $1.366(0.610-3.060)$ & 0.448 \\
\hline G3 & $1.724(0.746-3.985)$ & 0.202 & $1.857(0.688-5.012)$ & 0.222 \\
\hline Intravesical therapy (no vs. yes) & $3.089(1.556-6.132)$ & 0.001 & $3.794(1.680-8.570)$ & 0.001 \\
\hline miR-200a (low vs. high level) & $0.539(0.296-0.982)$ & 0.022 & $0.449(0.239-0.842)$ & 0.013 \\
\hline
\end{tabular}

HR, hazard risk; CI, confidence interval.

\section{Discussion}

The current study confirmed that miRNAs in urine supernatant can serve as diagnostic and prognostic biomarkers of bladder cancer. An ideal biomarker should be easily accessible by non-invasive protocols, inexpensive to quantify, specific to the disease of interest, and a reliable indicator of disease before clinical symptoms appear (9). Recently, numerous studies have shown that miRNAs are emerging as a new class of cancer biomarkers (13). miRNAs have been identified in all types of body fluids, including plasma, tears, saliva, peritoneal fluid and urine (9). Of these candidate sources, urine is a particularly desirable source of biomarkers for bladder cancer. First, cell-free miRNAs in urine could be direct indicators of the urological condition. The exact mechanisms of release and the roles of cell-free circulating miRNAs are still largely unclear. Recent studies have suggested that the interaction between cells via mRNA and miRNA is accomplished by microvesicle transfer (14). Circulating miRNAs might be released from tumor cells in microvesicles as a mechanism to communicate with 
recipient cells in the surrounding microenvironment (15). If so, there is no doubt that urine would have the highest exposure to microvesicles from cancer tissue. Interestingly, the composition and concentrations of cell-free miRNAs are different in serum and urine $(9,16,17)$, which suggests that urinary cell-free miRNAs probably originate from the urinary tract per se rather than from plasma filtrate (17). In addition, urine is far more convenient and less invasive to collect than blood, and miRNAs exist in a stable form in urine. Even after seven cycles of freezing and thawing or $72 \mathrm{~h}$ at room temperature, miRNA levels in the urine were largely unchanged.

The current study demonstrated that miR-145 in urine could be a marker for bladder cancer. Levels of miR-145 could distinguish bladder cancer patients from the non-cancer controls $(77.8 \%$ sensitivity and $61.1 \%$ specificity for NMIBC; 84.1 and $66.1 \%$ for MIBC, respectively) and showed good correlation with grade. Although it did not have outstanding power as a single diagnostic marker, we believe its diagnostic accuracy for bladder cancer will be improved by use in combination with conventional markers such as urine cytology. miR-145 is known to function as a tumor suppressor by inducing apoptosis. The expression of exogenic miR-145 was shown to induce both caspase-mediated and caspase-independent apoptosis. The destabilization of mRNAs containing several miR-145 target sites was observed, and the direct regulation of CBFB, PPP3CA and CLINT1 has been demonstrated $(18,19)$. Several studies demonstrated that miR-145 levels in cancer tissue were decreased compared to the normal bladder mucosae (20-22), and notwithstanding the current study was conducted in urine sample, these results are in good agreement with our data.

In addition, miR-200a and intravesical chemotherapy were independent predictors of NMIBC recurrence by univariate and multivariate analysis. Kaplan-Meier analysis showed that patients with lower miR-200a in urine had a higher risk of recurrence of NMIBC than those with higher miR-200a. Several independent reports have implicated miR-200 family members in epithelial to mesenchymal transition (EMT), an important event in tumorigenesis that is associated with a decrease in E-cadherin levels, loss of cell adhesion and subsequent tumor invasion and metastasis (23-25). Gregory et al reported that the loss of miR-200 expression leads to the accumulation of ZEB1 and ZEB2, which is sufficient to silence CDH1 and promote EMT and tumor invasion (26). In terms of bladder cancer, a recent study suggested that EMT could play an important role in NMIBC recurrence (27). Given the evidence, we hypothesize that higher levels of urinary miR-200a might inhibit EMT and prevent NMIBC recurrence through the silencing of various target genes. To fully understand the relationship between miR200a expression and NMIBC recurrence, further functional evaluation will be needed.

To our knowledge, the current study is the first report to evaluate urinary cell-free miRNAs as diagnostic and prognostic biomarkers of bladder cancer. Only one previous study has reported the investigation of miRNAs in urine in relation to the stage and grade of bladder cancer (10). However, this study used the pellet obtained from centrifuged urine; thus, which miRNAs were not cell-free. Intracellular miRNAs may have distinct differences from those in urine or serum. Also, an uneven amount of cellular components, such as red blood cells, white blood cells and cancer cells, could influence the reproducibility as reliable biomarkers.

The current study indicates that cell-free miRNAs in urine offer promise as noninvasive biomarkers for diagnosing and predicting the prognosis of bladder cancer. miR-145 in urine represents a potential diagnostic biomarker for the onset of bladder cancer, while miR-200a appears to be a predictor of the recurrence of NMIBC.

\section{Acknowledgements}

The biospecimens for this study were provided by the Chungbuk National University Hospital, a member of the National Biobank of Korea, which is supported by the Ministry of Health, Welfare and Family Affairs. All samples were obtained with informed consent under institutional review board-approved protocols. This research was supported by the Korea Healthcare Technology R\&D Project, Ministry of Health \& Welfare, Republic of Korea (A100651-1011-0000100).

\section{References}

1. Van der Poel HG and Debruyne FM: Can biological markers replace cystoscopy? An update. Curr Opin Urol 11: 503-509, 2001.

2. Simon MA, Lokeshwar VB and Soloway MS: Current bladder cancer tests: unnecessary or beneficial? Crit Rev Oncol Hematol 47: 91-107, 2003

3. Giannopoulos A, Manousakas T, Gounari A, et al: Comparative evaluation of the diagnostic performance of the BTA stat test, NMP22 and urinary bladder cancer antigen for primary and recurrent bladder tumors. J Urol 166: 470-475, 2001.

4. Ambros V: The functions of animal microRNAs. Nature 431: 350-355, 2004.

5. Bartel DP: MicroRNAs: genomics, biogenesis, mechanism, and function. Cell 116: 281-297, 2004.

6. Calin GA, Sevignani C, Dumitru CD, et al: Human microRNA genes are frequently located at fragile sites and genomic regions involved in cancers. Proc Natl Acad of Sci USA 101: 2999-3004, 2004.

7. Lu J, Getz G, Miska EA, et al: MicroRNA expression profiles classify human cancers. Nature 435: 834-838, 2005.

8. Chen CZ: MicroRNAs as oncogenes and tumor suppressors. N Engl J Med 353: 1768-1771, 2005.

9. Weber JA, Baxter DH, Zhang S, et al: The microRNA spectrum in 12 body fluids. Clin Chem 56: 1733-1741, 2010.

10. Yamada Y, Enokida H, Kojima S, et al: MiR-96 and miR-183 detection in urine serve as potential tumor markers of urothelial carcinoma: correlation with stage and grade, and comparison with urinary cytology. Cancer Sci 102: 522-529, 2011.

11. Hanke M, Hoefig K, Merz $\mathrm{H}$, et al: A robust methodology to study urine microRNA as tumor marker: microRNA-126 and microRNA-182 are related to urinary bladder cancer. Urol Oncol 28: 655-661, 2010.

12. Meiri E, Levy A, Benjamin H, et al: Discovery of microRNAs and other small RNAs in solid tumors. Nucleic Acids Res 38: 6234-6246, 2010.

13. Jeffrey SS: Cancer biomarker profiling with microRNAs. Nat Biotechnol 26: 400-401, 2008.

14. Valadi H, Ekstrom K, Bossios A, et al: Exosome-mediated transfer of mRNAs and microRNAs is a novel mechanism of genetic exchange between cells. Nat Cell Biol 9: 654-659, 2007.

15. Skog J, Wurdinger T, van Rijn S, et al: Glioblastoma microvesicles transport RNA and proteins that promote tumour growth and provide diagnostic biomarkers. Nat Cell Biol 10: 1470-1476, 2008.

16. Gilad S, Meiri E, Yogev Y, et al: Serum microRNAs are promising novel biomarkers. PloS One 3: e3148, 2008.

17. Wang G, Tam LS, Li EK, et al: Serum and urinary cell-free MiR-146a and MiR-155 in patients with systemic lupus erythematosus. J Rheumatol 37: 2516-2522, 2010. 
18. Ostenfeld MS, Bramsen JB, Lamy $\mathrm{P}$, et al: miR-145 induces caspase-dependent and -independent cell death in urothelial cancer cell lines with targeting of an expression signature present in Ta bladder tumors. Oncogene 29: 1073-1084, 2010.

19. Schaefer A, Stephan C, Busch J, et al: Diagnostic, prognostic and therapeutic implications of microRNAs in urologic tumors. Nat Rev Urol 7: 286-297, 2010.

20. Ichimi T, Enokida H, Okuno Y, et al: Identification of novel microRNA targets based on microRNA signatures in bladder cancer. Int J Cancer 125: 345-352, 2009.

21. Dyrskjot L, Ostenfeld MS, Bramsen JB, et al: Genomic profiling of microRNAs in bladder cancer: miR-129 is associated with poor outcome and promotes cell death in vitro. Cancer Res 69: 4851-4860, 2009

22. Friedman JM, Liang G, Liu CC, et al: The putative tumor suppressor microRNA-101 modulates the cancer epigenome by repressing the polycomb group protein EZH2. Cancer Res 69: 2623-2629, 2009

23. Park SM, Gaur A, Lengyel E and Peter ME: The miR-200 family determines the epithelial phenotype of cancer cells by targeting the E-cadherin repressors ZEB1 and ZEB2. Genes Dev 22: 894-907, 2008.
24. Korpal M, Lee ES, Hu G and Kang Y: The miR-200 family inhibits epithelial-mesenchymal transition and cancer cell migration by direct targeting of E-cadherin transcriptional repressors ZEB1 and ZEB2. J Biol Chem 283: 14910-14914, 2008.

25. Bracken CP, Gregory PA, Kolesnikoff N, et al: A double-negative feedback loop between ZEB1-SIP1 and the microRNA-200 family regulates epithelial-mesenchymal transition. Cancer Res 68: 7846-7854, 2008.

26. Gregory PA, Bert AG, Paterson EL, et al: The miR-200 family and miR-205 regulate epithelial to mesenchymal transition by targeting ZEB1 and SIP1. Nat Cell Biol 10: 593-601, 2008.

27. Bruyere F, Namdarian B, Corcoran NM, et al: Snail expression is an independent predictor of tumor recurrence in superficial bladder cancers. Urol Oncol 28: 591-596, 2010. 